\title{
The 47th Annual Meeting of the European Society for Blood and Marrow Transplantation: statistical symposium-poster session (P188-P189)
}

Published online: 24 June 2021

C Springer Nature Limited 2021

\section{4-17 March, $2021 \bullet$ Virtual Meeting}

Copyright: Modified and published with permission from https://www.ebmt.org/annual-meeting

Sponsorship statement: Publication of this supplement is sponsored by the European Society for Blood and Marrow Transplantation. All content was reviewed and approved by the EBMT Committee, which held full responsibility for the abstract selections.

\section{Statistical Symposium Poster Session}

P188.

Evaluation of Machine Learning Algorithms Applicability for Graft Failure Prediction

Evgeny Bakin ${ }^{1}$, Oksana Stanevich ${ }^{1}$, Ekaterina Demchenko $^{2}$, Maria Vladovskaya ${ }^{1}$, Elena Morozova ${ }^{1}$, Ludmilla Zubarovskaya ${ }^{1}$, Ivan Moiseev ${ }^{I}$

${ }^{1}$ RM Gorbacheva Research Institute of Pediatric Oncology, Hematology and Transplantation, Pavlov University, Saint Petersburg, Russian Federation, ${ }^{2}$ Saint Petersburg State University of Aerospace Instrumentation (SUAI), Saint Petersburg, Russian Federation

Background: The recovery of donor hematopoiesis is the main goal of allogeneic HSCT. To date, there is no unambiguous understanding of the key factors affecting the success of an engraftment. It is of interest to assess the applicability of routinely collected anamnestic data, as well as blood composition (leukocytes, neutrophils, platelets etc.) for early prediction of graft failure.

Methods: For the development of the prediction algorithm, we generated and preprocessed a dataset representing the results of laboratory tests (with the total amount of $>50 \mathrm{k}$ units) of 1371 patients with the first alloHSCTs performed in the period from 2014 to 2020 at RM Gorbacheva Research Institute (CIC725). An anamnestic data (age, gender, diagnosis and type of transplantation), as well as parameters of leukocytes, neutrophils and platelets recovery rate (represented as an intercept and a slope of fitted linear models) were selected as input variables for various machine learning (ML) algorithms. Based on this set of predictors, a few classifiers were built, including logistic regression, SVM, GBM and random forest. A prediction quality for all of the approaches was checked using leave-one-out (LOO) cross-validation. For each time point, starting from day +15 after HSCT we built a ROC curve, representing a current balance between sensitivity and specificity.

Results: The study showed that the random forest algorithm has the best efficiency among the considered ML classifiers. For it, by the day +20 after a beginning of HSCT an AUC reaches a value of 0.86 (specificity $=0.7$, sensitivity $=0.85$ ). Thus, 10 days before the standard time point +30 , the fact of primary non-engraftment can be predicted with a high reliability. The most important predictors were an intercept and a slope for WBC dynamics, an HSCT type and a patient diagnosis.

Conclusions: The study showed a general possibility of predicting primary graft failure basing on routinely collected data by the 20th day of a post-transplant period. This information can then be used to initiate preparation for a consequent second transplantation (or a boost). The developed tool can also be adapted for prediction of other clinically significant events basing on dynamically changing parameters of a patient.

Disclosure: Nothing to declare. 
P189.

\section{White Matter Development in Neonates With Infantile Krabbe Disease}

\section{Ashley Whited ${ }^{1}$, Keith Werling ${ }^{I}$, Michele Poe ${ }^{I}$, Maria Escolar $^{I}$}

${ }^{1}$ University of Pittsburgh, Pittsburgh, United States

Background: Infantile Krabbe disease (KD) is a rare neurodegenerative disorder characterized by progressive dysmyelination and demyelination. Initial symptoms can present with excessive irritability and difficulty nursing, progressing as the patient experiences seizures, vision loss, muscle spasms and cognitive impairment. Eventually most patients enter a vegetative state and death occurs-typically within 2 years of age. Previous studies have shown that diffusion measures like fractional anisotropy (FA) can accurately detect white matter degeneration in KD patients and guide clinical intervention in the form of hematopoietic stem cell transplantation (HSCT). As more advanced therapies (including gene therapy) are emerging, there is an increased need to map the development of this disease to identify and match disease progression biomarkers with treatments that provide the best outcomes for individual patients.

Methods: With the use of an automated diffusion tensor imaging (DTI) analysis pipeline, white matter structure was quantified using FA. Four major brain tracts (left and right corticospinal internal capsule (CSIC) and the splenium and genu of the corpus callosum) were analyzed. Volumetric registration of the FA maps for patients against appropriately age-matched atlases was used to align white matter structures. Successful registration was visually affirmed for quality assurance purposes. Automated fiber-tract quantification (AFQ) for FA was then performed for the four tracts listed above, and the average FA curves were compared to those of age-matched controls.

Results: Changes in white matter were recorded in 92 patients: 62 early infantile KD patients $(<12$ months at onset), 21 late infantile $\mathrm{KD}$ patients (12-36 months at onset), 5 healthy KD heterozygotes and 4 children that were identified as high risk through newborn screening. By 2 years of age, KD patients had FA values more than $50 \%$ below those of the healthy controls across the tracts with rapid decline in the first 6 months. Patients were sorted into high and low baseline FA groups and Kaplan-Meier survival curves indicated that early infantile KD patients with high FA values for the CSIC tract had a median survival 1 year longer than patients with low FA values. The baseline FA values of the splenium and the genu were not found to be associated with survival. None of the late infantile KD baseline FA values were found to be significantly associated with survival.

Conclusions: FA is a sensitive metric for assessing the progression of infantile $\mathrm{KD}$ and predicting therapeutic outcomes in HSCT candidates. If infantile KD is detected through newborn screening, DTI can be used to monitor changes in asymptomatic children at high risk for KD and maximize HSCT effectiveness through early diagnosis.

Disclosure: Nothing to declare. 